EDITORIAL

\title{
Nutrición en la prevención y el tratamiento de la obesidad: por fin nos hemos puesto todos de acuerdo
}

\section{Nutrition in the prevention and treatment of obesity: at last we all agree}

\author{
Jordi Salas-Salvadó* \\ Unidad de Nutrición Humana, IISPV, Universitat Rovira i Virgili, Reus, Tarragona, y CIBER Obesidad y Nutrición, \\ Instituto Carlos III, Madrid, España
}

Recibido el 13 de diciembre de 2011; aceptado el 21 de diciembre de 2011

Es para mí un placer invitar a los dietistas-nutricionistas y demás profesionales de la salud que reciben periódicamente Revista Española de Nutrición Humana y Dietética a que lean el documento de consenso "Recomendaciones nutricionales basadas en la evidencia para la prevención y el tratamiento del sobrepeso y la obesidad en adultos"'. Este importante documento, en el que he tenido el honor de participar como coordinador junto al Dr. Xavier Formiguera Sala, ha sido elaborado por la Federación Española de Sociedades de Nutrición, Alimentación y Dietética (FESNAD) (de la que forma parte la Asociación Española de Dietistas-Nutricionistas [AED-N]), a iniciativa de y conjuntamente con la Sociedad Española para el Estudio de la Obesidad (SEEDO). Se trata de un consenso que ha revisado la bibliografía científica publicada entre el 1 de enero de 1996 y el 31 de enero de 2011, relacionada con las áreas de interés general del documento. Las conclusiones obtenidas tras dicha revisión se clasificaron según el método propuesto en 2008 por la Asociación Europea para el Estudio de la Obesidad ${ }^{2}$, que consiste en una versión simplificada del sistema propugnado por la Scottish Intercollegiate Guidelines Network (SING) (Red Escocesa Intercolegiada sobre Guías de Práctica Clínica) ${ }^{3}$. Es importante recalcar que el documento refleja opiniones consensuadas entre los representantes de las distintas sociedades referidas en la autoría y, por lo tanto, constituye la postura de

\footnotetext{
*Autor para correspondencia.

Correo electrónico: jordi.salas@urv.cat (J. Salas-Salvadó).
}

todas ellas. Asimismo, ha contado con la desinteresada revisión realizada por acreditados expertos en el campo de la obesidad (Dres. Juan María Ballesteros, Miguel Ángel Rubio, Miguel Ángel Martínez y José María Ordovás) y la colaboración de la Agencia Española de Seguridad Alimentaria y Nutrición (AESAN). Al ser un documento de acceso gratuito desde diferentes páginas web (FESNAD, SEEDO, GREPAED-N, SENPE, AESAN), se espera una amplia difusión.

Se trata de un documento que complementa anteriores consensos españoles relacionados con la obesidad ${ }^{5} \mathrm{y}$, desde mi punto de vista, absolutamente necesario, ya que la obesidad (que sin duda debemos clasificar como "enfermedad"') está sustituyendo a otros problemas que tradicionalmente eran los principales causantes de la pérdida de salud, como las enfermedades infecciosas o la desnutrición ${ }^{7,8}$. Como las tasas de mortalidad aumentan con el exceso de peso medido en función del índice de masa corporal (IMC) ${ }^{9}$, no es de extrañar que recientemente la Organización Mundial de la Salud (OMS) haya estimado que aproximadamente 2,8 millones de personas mueren al año en el mundo como resultado del sobrepeso o la obesidad ${ }^{8}$. Esto se debe, entre otros factores, a que el aumento del IMC poblacional se acompaña de un incremento en la proporción de personas con una o más comorbilidades ${ }^{10}$. Así, el aumento de la prevalencia de obesidad se ha visto acompañado por un incremento espectacular de la prevalencia de diabetes mellitus (DM) tipo $2^{8,11}$. Un reciente metaanálisis concluyó, tras combinar doce estudios prospectivos de cohortes homogéneos y de alta calidad, que la obesidad multiplica por 7 el riesgo 
de padecer DM2 ${ }^{12}$. Además de la DM, otras comorbilidades que se asocian a la obesidad son las enfermedades del corazón, los accidentes cerebrovasculares, la hipertensión o la dislipemia ${ }^{7,8}$, razón por la cual determinados expertos se refieren a los riesgos para la salud asociados a la obesidad como un "tsunami cardíaco"13. También incrementa el riesgo de padecer ciertos tipos de cáncer, enfermedad de la vesicula biliar, osteoartritis, gota y enfermedades pulmona$\mathrm{res}^{7,8,14}$. La persona obesa también sufre mayor riesgo de discriminación, estigma o prejuicio social, no sólo por los individuos de su entorno, sino incluso de los profesionales sanitarios, lo cual, paradójicamente, influirá negativamente en su búsqueda de ayuda médica ${ }^{7,15}$.

Como vemos, la obesidad supone una creciente amenaza para la salud de la población y es por ello que este consenso llega en el momento oportuno. Estoy seguro de que estamos, tal y como señala el Dr. Roberto Sabrido (presidente de la $A E S A N$ ) en el prólogo de este consenso, ante "una herramienta de gran utilidad para el profesional sanitario, que le permitirá prescribir dietas basándose en el mejor conocimiento existente y contestar, con exactitud, a las numerosas preguntas que seguramente recibe en la consulta y fuera de la misma".

Aprovecho para agradecer en nombre de la FESNAD y la SEEDO al Comité de Redacción del Documento, que ha sido liderado por profesionales de diferentes orígenes sanitarios, sus ejemplares dedicación, rigor y capacidad en consensuar. También a Nutrition et Santé agradecemos que facilitara la logística necesaria para su realización, respetando en todo momento la independencia de criterio de los redactores y revisores.

\section{Bibliografía}

1. Gargallo Fernández M, Basulto Marset J, Breton Lesmes I, Quiles Izquierdo J, Formiguera Sala X, Salas-Salvadó J. Recomendaciones nutricionales basadas en la evidencia para la prevención y el tratamiento del sobrepeso y la obesidad en adultos (Consenso FESNAD-SEEDO). Rev Esp Obes. 2011;9 Supl 1:1-78.

2. Tsigos C, Hainer V, Basdevant A, Finer N, Fried M, Mathus-Vliegen $\mathrm{E}$, et al. Management of obesity in adults: European clinical practice guidelines. Obes Facts. 2008;1:106-16.
3. Scottish Intercollegiate Guidelines Network. A guideline developers' handbook (Publication $n^{\circ}$ 50). Edinburgh: SIGN; 2001 [monografía en internet] [actualizado 1 Ene 2008; citado 15 Ene 2011]. Disponible en: http://www.sign.ac.uk/guidelines/ fulltext/50/index.html

4. Arrizabalaga JJ, Masmiquel L, Vidal J, Calañas-Continente A, Díaz-Fernández MJ, García-Luna PP, et al; Grupo de Trabajo sobre Obesidad de la Sociedad Española de Endocrinología y Nutrición. Recomendaciones y algoritmo de tratamiento del sobrepeso y la obesidad en personas adultas. Med Clin (Barc). 2004;122:104-10.

5. Salas-Salvadó J, Rubio MA, Barbany M, Moreno B; Grupo Colaborativo de la SEEDO. Consenso SEEDO 2007 para la evaluación del sobrepeso y la obesidad y el establecimiento de criterios de intervención terapéutica. Med Clin (Barc). 2007;128:18496.

6. Basulto J, Manera M, Baladia E. Postura del GREP-AEDN: la obesidad como enfermedad. Act Diet. 2008;12:98-9.

7. World Health Organization. Obesity: preventing and managing the global epidemic. Report of a WHO consultation. World Health Organ Tech Rep Ser. 2000;894:1-253.

8. World Health Organization. Global status report on noncommunicable diseases. Geneva: WHO Library Cataloguing-in-Publication Data; 2011.

9. Berrington de Gonzalez A, Hartge P, Cerhan JR, Flint AJ, Hannan L, Maclnnis RJ, et al. Body-mass index and mortality among 1.46 million white adults. N Engl J Med. 2010;363:2211 . 9.

10. Flegal KM, Graubard BI, Williamson DF, Gail MH. Excess deaths associated with underweight, overweight, and obesity. JAMA. 2005;293:1861-7.

11. Mokdad AH, Bowman BA, Ford ES, Vinicor F, Marks JS, Koplan JP. The continuing epidemics of obesity and diabetes in the United States. J Am Diet Assoc. 2001;286:1195-200.

12. Abdullah A, Peeters A, De Courten M, Stoelwinder J. The magnitude of association between overweight and obesity and the risk of diabetes: a meta-analysis of prospective cohort studies. Diabetes Res Clin Pract. 2010;89:309-19.

13. Anand SS, Yusuf S. Stemming the global tsunami of cardiovascular disease. Lancet. 2011;377:529-32.

14. World Cancer Research Fund/American Institute for Cancer Research. Food, nutrition, physical activity, and the prevention of cancer: a global perspective. Washington: AICR; 2007.

15. Brownell KD, Kersh R, Ludwig DS, Post RC, Puhl RM, Schwartz $M B$, Willett WC. Personal responsibility and obesity: a constructive approach to a controversial issue. Health Aff (Millwood). 2010;29:379-87. 